\title{
Hommage à Alexandre Favre
}

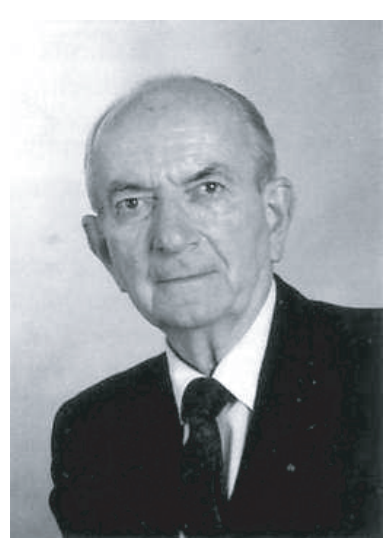

Le Mercredi 25 mai, Alexandre Favre, Professeur émérite à l'Université de la Méditerranée, membre de l'Académie des Sciences, s'est éteint à l'âge de 94 ans.

En 1932, dès la fin de ses études à l'École d'ingénieurs de Marseille et à la faculté des sciences de Marseille, A. Favre décide de se consacrer à l'enseignement supérieur et la recherche scientifique dans une discipline alors en plein essor : la mécanique des fluides. Recruté comme assistant et affecté à l'institut de mécanique des fluides de Marseille, récemment créé dans le cadre d'une convention entre le Ministère de l'Air et les universités, il y prépare son doctorat ès sciences physiques, qu'il soutient en 1938. Il franchit ensuite toutes les étapes de la carrière universitaire. Successivement chef de travaux, chargé puis maître de conférences, professeur sans chaire, il est nommé en 1951 professeur titulaire de la chaire de mécanique de l'atmosphère et météorologie à la faculté des sciences de Marseille, puis à l'université d'Aix-Marseille II jusqu'à sa retraite en 1980. En 1962-1963, il est visiting professor à l'université Johns Hopkins de Baltimore. Depuis 1981, il demeurait professeur émérite de l'université de la Méditerranée.

A. Favre a toujours participé très activement à la vie de l'université, et consacré beaucoup de son temps aux innombrables conseils dont il a fait partie. Son ouvre majeure au plan universitaire restera évidemment la fondation en 1960 de l'institut de mécanique statistique de la turbulence, dont il a été directeur durant vingt ans. Nous y reviendrons. Il était très bon pédagogue, et ses cours étaient des modèles de précision et de clarté. À l'université, ils concernaient surtout la turbulence et révélaient sa passion pour le sujet : c'est dans ce cadre qu'il a progressivement mis au point sa fameuse « méthode $B »$ qui a conduit aux «moyennes de Favre». Les exigences de précision et de rigueur sont également celles qu'il a su inculquer aux générations d'étudiants qui se sont succédées dans son laboratoire pour des stages ou des thèses.
Il n'a pas oublié qu'il était issu (promotion 1931) de l'École d'ingénieurs de Marseille. Il est resté très attaché à son école, où il a enseigné la mécanique des fluides durant près de 30 ans et recruté de nombreux élèves et collaborateurs. En avance sur son temps, c'était un partisan convaincu du rapprochement entre écoles d'ingénieurs et universités : dans les années 70, il a soutenu un projet de «nationalisation » de l'E.I.M.

Ses premiers travaux concernent la mécanique des fluides et l'aérotechnique. Son étude expérimentale des mouvements hydrodynamiques lui fait imaginer durant sa thèse de supprimer les effets de couche limite en déplaçant la surface de l'obstacle à la vitesse du fluide : c'est l'« aile à paroi mobile » hypersustentatrice. Affecté en 1939-1940 à la Société nationale de constructions de moteurs, il y conçoit un nouveau type de compresseur centrifuge supersonique à diffuseurs séparés. En raison des circonstances, il dissimule et transmet aux alliés cette invention qui a été utilisée dans les premières générations de turboréacteurs britanniques et, plus tard, à l'usine de séparation isotopique de Pierrelatte.

C'est peu après qu'il prend la décision capitale de se consacrer désormais à l'étude de la turbulence des écoulements fluides. Il a pour cela une idée originale : étudier leur structure par des mesures statistiques en deux points séparés non seulement dans l'espace mais aussi dans le temps. C'est la méthode des « corrélations spatio-temporelles ». Avec le soutien du G.R.A., puis de l'O.N.E.R.A., il rassemble une équipe, fait construire des appareils, et monte de toutes pièces un laboratoire. Son idée directrice est que l'étude expérimentale d'une grande variété d'écoulements turbulents mettra en évidence des caractéristiques communes qui permettront d'en éclaircir le mystère. Aux recherches soutenues par l'O.N.E.R.A pour l'aéronautique subsonique viennent s'ajouter celles faites pour le C.E.A en vue de la séparation isotopique, puis l'O.N.E.R.A. demande (c'est l'époque du projet Concorde) que l'on s'attaque aux problèmes très ardus de la turbulence en supersonique. Il va falloir pour cela construire un équipement lourd sans aucun équivalent : une soufflerie supersonique à faible niveau de turbulence.

C'est l'occasion pour A. Favre de faire aboutir un projet qu'il caressait depuis quelques années : la création d'un institut entièrement consacré aux recherches sur la turbulence. Il réussit à convaincre les structures de tutelle (la direction des enseignements supérieurs via l'université, et le Centre National de la Recherche Scientifique) qui s'intéressaient depuis déjà longtemps à ses travaux, et bien d'autres organismes et personnalités scientifiques, et obtient en 1960 la création de l'Institut de 
Mécanique Statistique de la Turbulence. Le sigle I.M.S.T. est bientôt connu dans tout le monde scientifique, et le laboratoire constitue désormais un lieu de passage obligé pour les spécialistes de la discipline de tous les pays. Le développement de l'Institut, désormais U.E.R. à dérogation de l'Université et Laboratoire Associé au C.N.R.S. se poursuit alors. Dès 1970, il faut créer et faire fonctionner sur le campus de Luminy, avec le soutien de la D.G.R.S.T. et du C.N.E.X.O., puis d'E.D.F. et d'autres organismes, une annexe dotée d'un autre équipement alors unique au monde : une grande soufflerie de simulation des échanges Océan-Atmosphère.

Dans la fondation et la direction de l'I.M.S.T., A. Favre a fait preuve d'une volonté et d'une persévérance sans failles pour vaincre les multiples obstacles rencontrés. Il a réussi à rassembler autour de lui une équipe de collaborateurs entre lesquels il a su faire régner l'harmonie. Son seul véritable intérêt était la recherche fondamentale, mais il a su nouer - d'abord personnellement, puis à travers ses collaborateurs - des collaborations étroites et durables avec les grands organismes à finalité industrielle. Il possédait enfin une capacité exceptionnelle à nouer des relations scientifiques avec les autres chercheurs de la discipline. Ayant compris très tôt qu'une bonne recherche devait être internationale, il a effectué de nombreux séjours à l'étranger, surtout aux États-Unis. Il y recueillait de précieuses informations et y établissait des collaborations scientifiques doublées de relations amicales. Sur ce dernier plan, il a été remarquablement soutenu par son épouse, Luce Favre, avec laquelle il formait un couple très uni, qui a élevé trois enfants. Un de ses grands succès a été l'organisation en 1961, pour l'inauguration de l'I.M.S.T., de deux colloques internationaux sur la turbulence. Il est parvenu à y rassembler les plus éminents spécialistes vivant à l'époque, notamment des représentants de l'école soviétique conduits par le grand A.N. Kolmogorov qui a révélé à cette occasion sa célèbre « troisième hypothèse » sur l'intermittence interne de la turbulence.

A. Favre était doué d'un remarquable sens physique. Il est l'auteur - ou le co-auteur avec ses collaborateurs - d'un livre sur la turbulence, de près d'une centaine de publications scientifiques, et d'un grand nombre de communications à des congrès, conférences et rapports, sur les aspects les plus divers de la turbulence des écoulements fluides. Sans tenter ici d'en faire une synthèse exhaustive, nous citerons les deux thèmes sur lesquels il a le plus travaillé. Le premier est l'exploration fine de la structure interne des écoulements turbulents, et la mise en évidence de leur forte «mémoire » dans un repère à peu près lié au mouvement moyen, grâce à la méthode des corrélations spatio-temporelles. Devenue classique, cette méthode a permis l'identification d'une organisation semi-déterministe au sein du chaos turbulent, et a ainsi contribué à la mise au point des « méthodes de fermeture » couramment utilisées de nos jours par l'industrie dans les modèles numériques. Le second est l'emploi des «moyennes pondérées par la masse » pour écrire les équations statistiques de la turbulence. A. Favre a réalisé dès les années 50 que ces moyennes, proposées par certains météorologistes, étaient fondamentalement supérieures aux traditionnelles « moyennes de Reynolds » dans tous les cas où la masse spécifique du fluide subissait des fluctuations significatives. Il les a étudiées dans les moindres détails, et s'est fait l'avocat de leur emploi dans divers domaines (forts transferts de chaleur ou de masse, combustion, écoulements compressibles) où les «moyennes de Favre » sont aujourd'hui couramment utilisées.

A. Favre a continué à travailler, notamment pour l'Académie des Sciences, durant ses années de retraite. Avec quatre collègues de l'Institut de France, il a écrit et publié un livre de réflexions philosophiques sur la causalité et la finalité, où transparaissent ses profondes convictions religieuses.

Ce panorama de ses travaux serait incomplet s'il omettait les multiples et souvent lourdes responsabilités qu'il a assumées dans les conseils et comités auxquels il a participé, ou qu'il a présidés, en France et à l'étranger. On pourrait citer le Comité Consultatif des Universités, le Comité National de la Recherche Scientifique, le Comité National Français de Mécanique. Nous mentionnerons tout spécialement ici les Associations Universitaires de Mécanique. Il a été l'un des fondateurs de l'Association Universitaire de Mécanique des Fluides, et a présidé l'A.U.M.F. puis la F.U.M.

Différentes distinctions témoignent des mérites et du rayonnement de A. Favre. Il est Officier de la Légion d'Honneur et de l'Ordre National du Mérite, Commandeur des Palmes Académiques, membre de l'Académie des Sciences, Arts et Lettres de Marseille. Successivement lauréat puis correspondant de l'Académie des Sciences, il y est élu comme membre en 1975. Le Professeur Favre restera pour ceux qui l'ont connu un grand enseignant, chercheur, ingénieur et administrateur, et un homme d'une grande perspicacité et d'une ténacité exceptionnelle.

Michel Coantic, le 14/10/2005. 